\title{
On a small collection of Swedish Lepidoptera, kept at the National Museum of Natural History, Sofia
}

\author{
Stanislav Abadjiev ${ }^{1}$, Stoyan Beshkov ${ }^{2}$
}

National Museum of Natural History, Bulgarian Academy of Sciences, 1 Tsar Osvoboditel Blvd, 1000 Sofia, Bulgaria, [1] abadjiev@nmnhs.com, [2] beshkov@nmnhs.com

\begin{abstract}
The paper presents an inventory of 28 species of butterflies and moths collected in the northernmost part of Sweden during 1931-1932. The collection is of certain historical value and is kept at the National Museum of Natural History at the Bulgarian Academy of Sciences, Sofia.
\end{abstract}

Keywords: butterfly, Lepidoptera, Luleå, moth, Sweden

\section{Introduction}

The National Museum of Natural History at the Bulgarian Academy of Sciences in Sofia is known as a depository of numerous and very important materials of butterflies and moths from the Balkan Peninsula, especially from Bulgaria. The old museum holdings are also rich in material from other regions, mainly with South American, African, Asian and European origin. Other than the Balkans, the European material is represented primarily by Lepidoptera collected in the countries of Central Europe. Isolated small collections, like a particular one from northernmost part of Scandinavia, also exist. The latter is dated from the first half of the last century. All specimens have been collected by Rudolf Rangnow during 1931-1932 near the town of Luleå, Norrbotten, Sweden.

\section{Results}

The collection consists of 63 specimens, associated with 28 species. Families are arranged according to van Nieukerken at al. (2011). Sequence and nomenclature of family Noctuidae follow Fibiger et al. (2010) with incorporated subsequent changes from recent taxonomic revisions. Each entry includes the taxon's name; the name on the label, if different from the previous one, enclosed in square brackets "[ ]"; date and specimens, as well as notes on subsequent publications and illustrations. If a text of a label is quoted each line of the label's text is separated by a vertical line "|".

\section{Hesperiidae}

- Pyrgus centaureae (Rambur, [1839]): 15.VI.1931 -1 đ̃; 19.VI.1931 - 1 ㅇ․

Lycaenidae

- Plebeius (Vacciniina) optilete (Knoch, 1781): VI.1931 - 2 ふぶ.

\section{Nymphalidae}

- Oeneis norna norna (Thunberg, 1791) (Fig. 1): 15.VI.1932 - 1 ふ̊; VI.1932 - 1 o.

- Erebia ligea (Linnaeus, 1758): VI.1931 - 1 o, 1 ㅇ.

- Erebia embla embla (Thunberg, 1791) [Erebia diesa [sic]]: VI.1931 - 1 ふै, 1 ․

- Erebia disa disa (Thunberg, 1791): 15.VI.1932 - 1 ô [Erebia embla Thnbg]; 16.VI.1932 - 1 ㅇ [Erebia diesa [sic]].

- Aglais urticae urticae (Linnaeus, 1758): VI.1931 $-1 \hat{\jmath}, 1$.

- Boloria (Clossiana) euphrosyne euphrosyne (Linnaeus, 1758): 15.VI.1932 - 1 o; 16.VI.1932 1 ‥ 


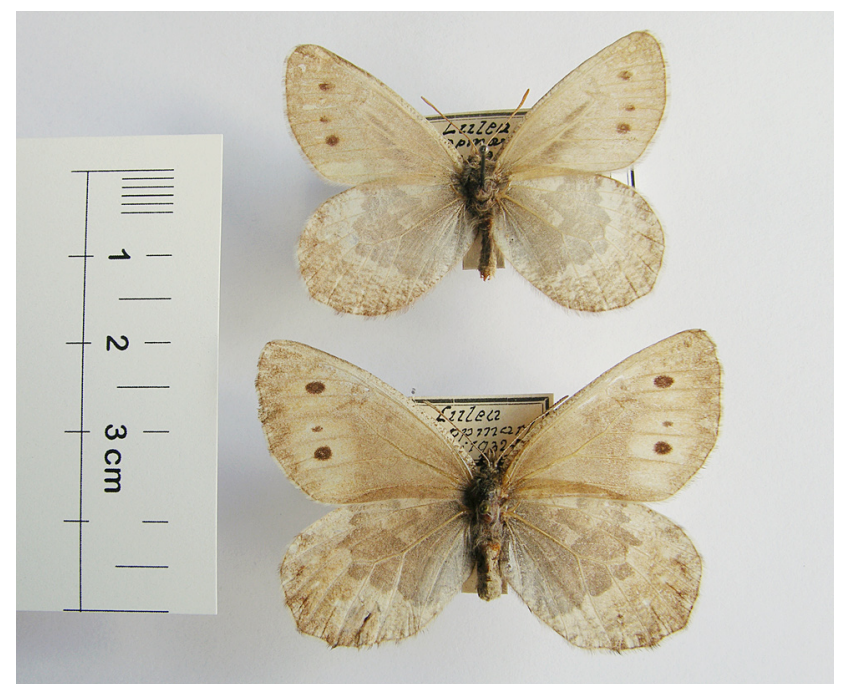

Fig. 1. Oeneis norna norna (Thunberg, 1791), male [above], female [below].

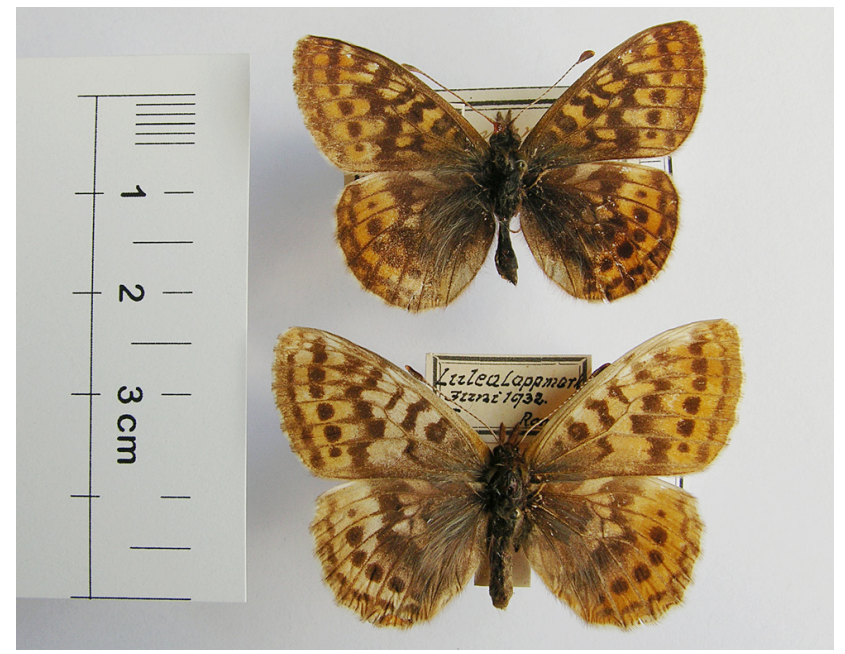

Fig. 2. Boloria (Clossiana) frigga frigga (Thunberg, 1791), male [above], female [below].

- Boloria (Clossiana) selene selene ([Denis \& Schiffermüller], 1775): 9.VI.1931 - 1 ठ̊; 16.VI.1932 1 ㅇ.

- Boloria (Clossiana) frigga frigga (Thunberg, 1791) (Fig. 2): VI.1932 - 2 के ठ, 2 우요.

- Boloria (Boloria) aquilonaris (Stichel, 1908) [Argynnis pales v. lappona [sic] Stgr.]: VI.1932 -

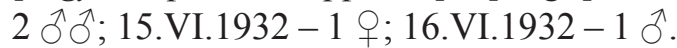

\section{Geometridae}

- Xanthorhoe abrasaria (Herrich-Schäffer, [1855]) [Larentia abrasaria H.S.]: VI.1931 - 1 ภ, 1 ㅇ.

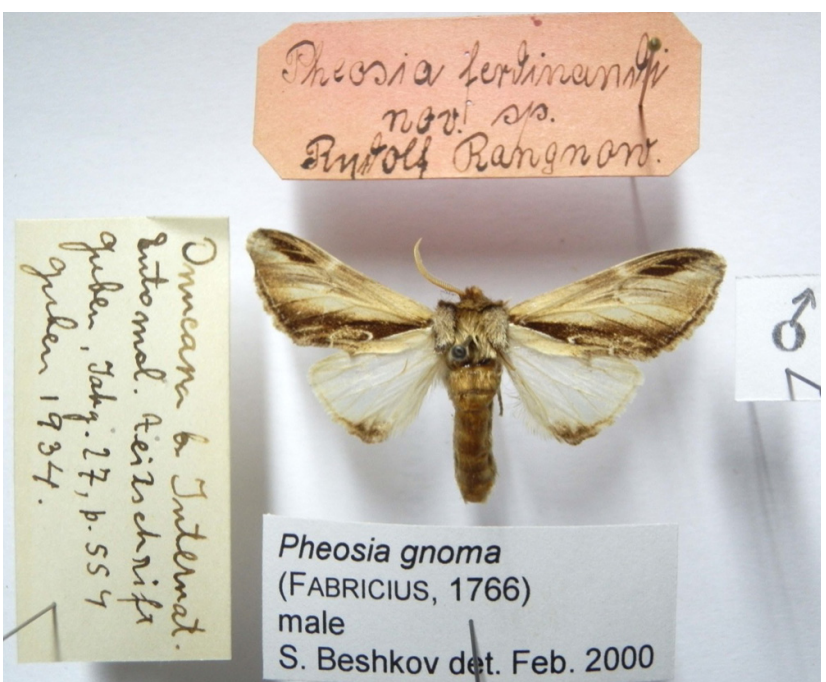

Fig. 3. Holotype of Pheosia ferdinandi Rangnow, 1934, a taxon identical with Pheosia gnoma (Fabricius, [1777]).

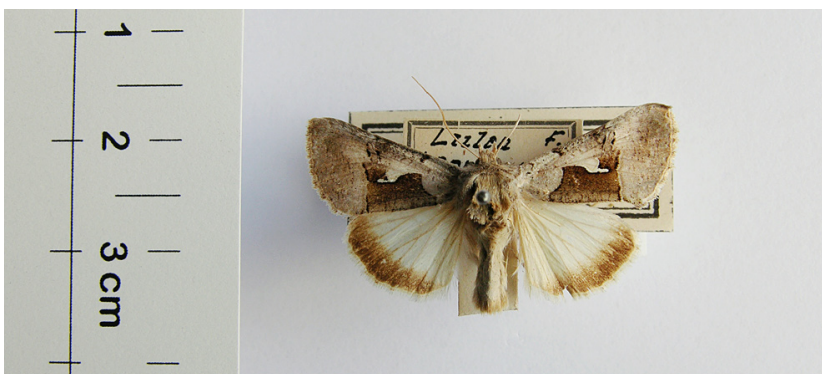

Fig. 4. Syngrapha diasema (Boisduval, 1829), male.

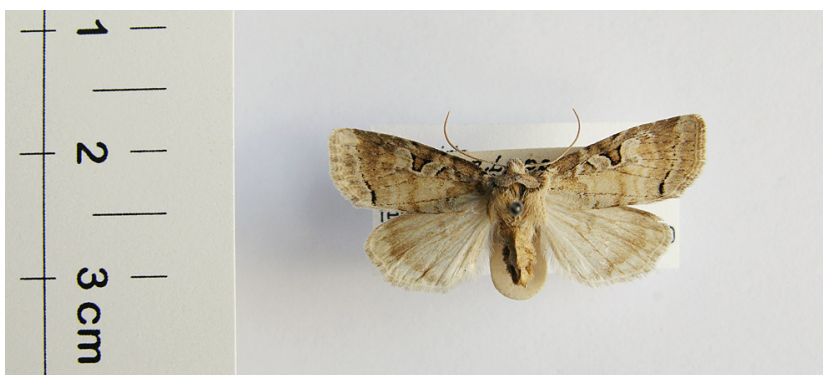

Fig. 5. Hillia iris (Zetterstedt, 1839), female.

- Heterothera serraria (Lienig, 1846) [Larentia serraria Z.]: VI.1931 - 1 ô, 1 \%.

\section{Notodontidae}

- Pheosia gnoma (Fabricius, [1777]) - 1 ठิ (Fig. 3) with labels: (1) handwritten (on red paper) "Pheosia ferdinandi | nov. sp. | Rudolf Rangnow"; (2) 
handwritten (on white paper) [by Dr Ivan Buresch] "Описана в [Described in] Internat. | Entomol. Zeitschrift | Guben, Jahrg. 27, p. 554 | Guben 1934."; (3) printed (on white paper) " ᄁᄁ"; (4) printed (on white paper) "Pheosia gnoma $\mid$ (FABRICIUS, 1766) $\mid$ male $\mid$ S. Beshkov det. Feb. 2000". The taxon has been described by Rangnow (1934: 555). It is identical with the species Pheosia gnoma (Fabricius, [1777]) (Schintlmeister, 1989: 91). The specimen represents a holotype (by monotypy) (ICZN, 1999: 73 a II) and is also illustrated in the original publication (Rangnow, 1934: 555).

\section{Noctuidae}

- Autographa macrogamma (Eversmann, 1842) [Plusia macro-gamma Ev.]: VI.1931 - 1 o; VI.1932 - 1 o. Published as Phytometra macrogamma Ev. nigroviolacea f. nov. (Rangnow, 1935: 22); Phytometra macrogamma Ev. interrupta f. nov. (Rangnow, 1935: 22); illustrated as Phytometra macrogamma nigroviolacea Rngn. (Rangnow, 1935: 21; Pl. 4: Fig. 6); Phytometra macrogamma interrupta Rngn. (Rangnow, 1935: 21; P1. 4: Fig. 7).

- Syngrapha parilis (Hübner, [1809]) [Plusia parilis Hb.]: VI.1932 - 1 ôं; VI.19311 - Q.

- Syngrapha diasema (Boisduval, 1829) (Fig. 4) [Plusia diasema B.]: VI.1932 - 1 đ̂; VI.1932 1 ․

- Syngrapha interrogationis (Linnaeus, 1758) [Plusia interrogationis L.]: 9.VI.1931 - 2 웅. Published as Syngrapha interrogationis L. magnifica f. nov. (Rangnow, 1935: 22). Illustrated as Syngrapha interrogationis magnifica Rngn. (Rangnow, 1935: 21; P1. 4: Fig. 9).

- Sympistis funebris (Hübner, [1809]) [Anarta funebris Hb.]: VI.1931 - 1 ô, 1 q (funesta Paykull, 1793 is a homonym of funesta Esper, [1786]). Published as Sympistis funesta Payk. nigrofasciata f. nov. (Rangnow, 1935: 21). Illustrated as Sympistis funesta nigrofasciata Rngn. (Rangnow, 1935: 21; P1. 4: Figures 1-2 [1- ô, 2 - q].

- Sympistis heliophila (Paykull, 1793) [Anarta melaleuca Thnbg.] (homonym: Noctua melaleuca Thunberg, 1791, nec Vieweg, 1790): VI.1931 1 ô, 1 ‥ Published as Sympistis melaleuca Thnbg. trimacula f. nov. (Rangnow, 1935: 22); Sympistis melaleuca Thnbg. leucofasciata f. nov. (Rangnow, 1935: 22); Sympistis melaleuca Thnbg. nigricata f. nov. (Rangnow, 1935: 22). Illustrated as Sympistis melaleuca trimacula Rngn. (Rangnow, 1935:
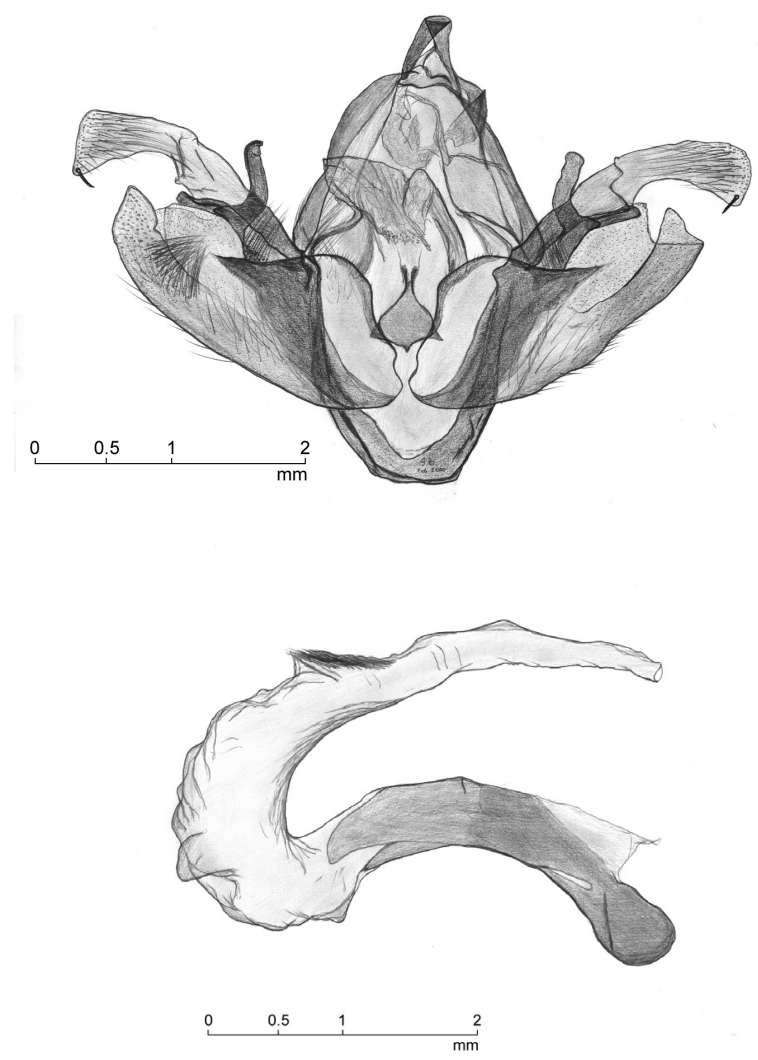

Figs 6-7. Polia lamuta (Herz, 1903), male genitalia with everted vesica.

21; Pl. 4: Fig. 3); Sympistis melaleuca leucofasciata Rngn. (Rangnow, 1935: 21; Pl. 4: Fig. 4); Sympistis melaleuca nigricata Rngn. (Rangnow, 1935: 21; P1. 4: Fig. 5).

- Hillia iris (Zetterstedt, 1839) (Fig. 5): VI.1931 $2 \hat{\jmath} \hat{0}, 2$ 우. Although all the labels clearly say "Juni", the month given here is most probably erroneous. The imago flies from the end of July to the end of August (Bengt $\AA$. Bengtsson, pers. comm.).

- Hadula melanopa melanopa (Thunberg, 1791) [Anarta melanopa Thnbg.]: VI.1931 - 1 Jे, 1 ․ .

- Coranarta cordigera cordigera (Thunberg, 1788) [Anarta cordigera Thnbg.]: VI.1931 - 2 วิ సิ.

- Polia lamuta (Herz, 1903) (Figs 6-7): 15.VI.1932 -1 ô, 1 q.

- Lasionycta secedens (Walker, 1858) [Anarta bohemanni (junior subjective synonym)]: VI.1931 -1 J, 1 O. Published as Polia bohemanni Stgr. unicolor f. nov. (Rangnow, 1935: 233); Polia bohemanni Stgr. nigrofasciata, f. nov. (Rangnow, 
1935: 233). Illustrated as Polia bohemanni Stgr. unicolor Rngn. (Rangnow, 1935: 224; Pl. 3: Fig. 32a); Polia bohemanni Stgr. nigrofasciata Rngn. (Rangnow, 1935: 224; P1. 3: Fig. 32b).

- Xestia tecta (Hübner, [1808]) [Agrotis tecta $\mathrm{Hb}$.$] :$

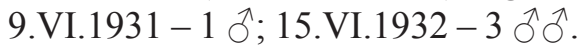

- Xestia speciosa arctica (Zetterstedt, 1839) [Agrotis speciosa Hb.]: 15.VI.1932 - 2 우. Published as Aplectoides speciosa arctica ab. nov. diffusa (Rangnow, 1935: 233). Illustrated as Aplectoides speciosa Hbn. arctica diffusa Rngn. (Rangnow, 1935: 224; P1. 3: Fig. 31).

- Xestia alpicola alpicola (Zetterstedt, [1839]) [Agrotis hyperborea Zett. (junior subjective synonym)]: VI.1931 - 1 §ో; VI.1932 - 1 §.

\section{Acknowledgments}

We are very much obliged to our colleagues Dr Boyan Zlatkov from the Institute of Biodiversity and Ecosystem Research, Sofia and Mr Bengt Å. Bengtsson, Färjestaden for their valuable suggestions and corrections.

\section{References}

Fibiger M., Ronkay L., Yela J.-L., Zilli A. 2010 Rivulinae-Phytometrinae, and Micronoctuidae, including Supplement to Noctuidae Europeae 1-11. Noctuidae Europeae 12: 1-450.

ICZN [International Commission on Zoological Nomenclature] 1999 International Code of Zoologi- cal Nomenclature. Fourth edition, adopted by the International Union of Biological Sciences. International Trust for Zoological Nomenclature, c/o The Natural History Museum, London, xxx+306 pp.

Rangnow R. 1934 Pheosia ferdinandi nov. sp. Internationale entomologische Zeitschrift 27 (48): 555.

Rangnow R. 1935 Lepidopterensammeln in Lappland. Entomologische Rundschau 52 (10): 133-136, (13): 175-176, (14): 188-190, (15): 202-205, (16): 223-224, (18): 233-234; 53 (2): 21-22.

Schintlmeister A. 1989 Zoogeographie der palaearktischen Notodontidae (Lepidoptera). Neue Entomologische Nachrichten 25: 1-117.

van Nieukerken E.J., Kaila L., Kitching I.J., Kristensen N.P., Lees D.C., Minet J., Mitter C., Mutanen M., Regier J.C., Simonsen T.J., Wahlberg N., Yen S.-H., Zahiri R., Adamski D., Baixeras J., Bartsch D., Bengtsson B.Å., Brown J.W., Bucheli S.R., Davis D.R., De Prins J., De Prins W., Epstein M.E., Gentili-Poole P., Gielis C., Hättenschwiler P., Hausmann A., Holloway J.D., Kallies A., Karsholt O., Kawahara A.Y., Koster S., Kozlov M.V., Lafontaine J.D., Lamas G., Landry J.-F., Lee S., Nuss M., Park K.-T., Penz C., Rota J., Schintlmeister A., Schmidt B.C., Sohn J.-C., Solis M.S., Tarmann G.M., Warren A.D., Weller S., Yakovlev R.V., Zolotuhin V.V., Zwick A. 2011 Order Lepidoptera Linnaeus, 1758. In: Zhang Z.-Q. (ed.) Animal biodiversity: An outline of higher-level classification and survey of taxonomic richness. Zootaxa 3148: 212-221. 